\title{
Mitigation of Engine Inlet Distortion through Adjoint-Based Design
}

\author{
Irian Ordaz*, Sriram K. Rallabhandi ${ }^{\dagger}$, Eric J. Nielsen ${ }^{\ddagger}$ \\ NASA Langley Research Center, Hampton, VA 23681 \\ and \\ Boris Diskin ${ }^{\S}$ \\ National Institute of Aerospace, Hampton, VA 23666
}

\begin{abstract}
The adjoint-based design capability in FUN3D is extended to allow efficient gradientbased optimization and design of concepts with highly integrated aero-propulsive systems. A circumferential distortion calculation, along with the derivatives needed to perform adjoint-based design, have been implemented in FUN3D. This newly implemented distortion calculation can be used not only for design but also to drive the existing mesh adaptation process and reduce the error associated with the fan distortion calculation. The design capability is demonstrated by the shape optimization of an in-house aircraft concept equipped with an aft fuselage propulsor. The optimization objective is the minimization of flow distortion at the aerodynamic interface plane of this aft fuselage propulsor.
\end{abstract}

\section{Introduction}

$\mathrm{T}$ HE aerospace industry continues to invest in research and novel technologies that promise improvements in aircraft efficiency. ${ }^{1,2}$ Aircraft efficiency can be quantified through a measure of engine fuel consumption. Innovative technologies for reduction of fuel consumption are being driven mainly by economic factors, such as the need to reduce operational costs. The close relationship between fuel consumption and emissions means that some technologies may also contribute to the research effort to meet future fuel and environmental regulations that seek to reduce the production of harmful emissions. In response, NASA has set goals to guide technology maturation for mid-term $(\mathrm{N}+2)$ and far-term $(\mathrm{N}+3)$ technology generations. ${ }^{3}$

Technologies that have contributed to past improvements in fuel efficiency include the use of light-weight structures, increased efficiency in components internal to the engine, and aerodynamic technologies associated with the wing (e.g. supercritical airfoils and winglets). ${ }^{4}$ However, there is currently growing interest in technologies that take advantage of the complex interaction between the airframe aerodynamics and the propulsion system in order to increase fuel efficiency. One technology in particular is boundary layer ingestion (BLI) ${ }^{5,6}$ which takes advantage of the low momentum flow from an ingested boundary layer (BL) to reduce engine ram drag and thus, the power required to produce thrust.

Competing performance objectives and a complex design space present challenges during the design of these highly integrated concepts. The benefits associated with BLI technologies come at the expense of reduced engine efficiency due to greater fan distortion and lower inlet efficiency. The design of an efficient inlet to optimize the complex flow field ingested by the engine is difficult and computationally expensive due to the higher order analysis needed to capture the physics of these problems. A significant concern is that flow distortion at the aerodynamic interface plane (AIP) can become severe and lead to increased vibration, noise, stress, and potentially greater likelihood of fan failure. The work of Gissen et al. ${ }^{7}$ demonstrated mitigation of distortion in a $5 \%$ scale model of a blended-wing-body by conditioning the flow upstream of the AIP. This

*Aerospace Engineer, Aeronautics Systems Analysis Branch, AIAA Member.

${ }^{\dagger}$ Aerospace Engineer, Aeronautics Systems Analysis Branch, AIAA Associate Fellow.

${ }^{\ddagger}$ Research Scientist, Computational AeroSciences Branch. AIAA Associate Fellow.

$\S$ NIA Research Fellow, AIAA Associate Fellow. 
mitigation was performed experimentally through the use of hybrid actuators including passive (vanes) and active (synthetic jets) technologies.

Traditional computational methods of computing gradients for design optimization, such as finite difference, are impractical for complex problems with a large set of design variables. As a result, the parametric space must be simplified or the problem must be decomposed into smaller subproblems which can produce suboptimal designs. The inherent sensitivity of real-valued finite difference methods to perturbation size can be resolved by the complex variable approach at the cost of greater number of floating point calculations. ${ }^{8}$

The adjoint method provides an efficient alternative for calculating design gradients of problems with a small number of objectives and constraints but large number of design variables. The application of a discrete adjoint approach to the BLI problem was successfully demonstrated by Lee et al. ${ }^{9}$ The formulation and implementation presented in this paper is similar to that earlier work where the distortion calculation is based on the SAE circumferential distortion descriptor (DPCP) defined by the Aerospace Recommended Practice (ARP) 1420 standard. $^{10}$ The distortion calculation used in Ref. 9 is based on flow samples along concentric rings on the engine AIP. In this paper, the distortion descriptor is represented by $D C_{\theta_{s}}$, where $\theta_{s}$ is the sector angle, and the implemented distortion calculation is based on the area- or mass-average of flow properties for all grid faces which lie on the AIP. This approach provides a more accurate representation of the flow distortion because it accounts for the contribution of all grid faces on the AIP. The work presented here is not intended to demonstrate the merits of BLI technologies but to apply discretely consistent adjoint-based sensitivity analysis to allow efficient gradient-based optimization of these highly integrated concepts.

The paper is organized into six sections. Following this introduction, Section II describes the FUN3D solver. Section III discusses the discrete adjoint formulation for the calculation of flow distortion at the AIP and corresponding sensitivity analysis for design. Section IV provides a verification of the sensitivity analysis. Section $\mathrm{V}$ discusses three demonstration cases: $i$ ) a demonstration of the mesh adaptation capability to reduce the error in the fan distortion calculation, ii) a verification case of the design optimization capability with a ducted fan geometry in freestream, and iii) a demonstration of the design optimization capability with a BLI geometry. Finally, a summary of the current and future work is provided in Section VI.

\section{FUN3D Solver}

Aerodynamic solutions and adjoint-based sensitivities are computed using FUN3D. ${ }^{11}$ FUN3D is a finitevolume node-centered unstructured-grid CFD solver, which is widely used for high-fidelity analysis and adjoint-based design of complex flows. FUN3D solves the governing flow equations on mixed-element grids containing arbitrary combinations of tetrahedra, pyramids, prisms, and hexahedra. At each control-volume face, the inviscid flux is computed using Roe's flux-difference splitting Riemann solver. For second-order accuracy, solution values at the face are obtained using a monotonic upstream-centered scheme for conservation laws (MUSCL) ${ }^{12}$ scheme, with unweighted least-squares gradients computed at the grid points. The viscous fluxes use the full viscous stress tensors. For tetrahedral meshes, the viscous fluxes are discretized using the GreenGauss element-based gradients; this is equivalent to a Galerkin-type approximation. For non-tetrahedral elements, the GreenGauss gradients are augmented with edge-based terms.

\section{Flow Distortion Objective Function}

The function $F$ is used to represent the flow distortion objective $D C_{\theta_{s}}$

$$
F(\boldsymbol{D}, \boldsymbol{X}, \boldsymbol{Q})=D C_{\theta_{s}}=\frac{\bar{P}_{t}-\bar{P}_{t, \theta_{s}}}{\bar{Q}}
$$

that depends on design parameters, $\boldsymbol{D}$; computational grid, $\boldsymbol{X}$; and solution, $\boldsymbol{Q}$. Specifically, $\bar{P}_{t}$ and $\bar{Q}$ are the mass-averaged total pressure and dynamic pressure, respectively, integrated over the entire AIP; $\bar{P}_{t, \theta_{s}}$ is the lowest mass-averaged total pressure integrated over a sector of the AIP with the sector angle $\theta_{s}$.

The discrete formulation and the corresponding implementation of the objective function defined in Eq. 1 are based on the node-centered unstructured-grid discretization of the Reynolds-Averaged Navier-Stokes (RANS) equations described in Ref. 13. The implementation relies on the comprehensive and verified sensitivity analysis for steady and unsteady flows computed on general static and dynamic, rigidly moving and deforming, single-block and overset grids. ${ }^{13,14}$

The objective function, Eq. 1, involves integration of weighted total and dynamic pressure within the 
AIP that coincides with the fan plane shown in Fig. 1. Area weighting and mass weighting options are implemented. Integration is conducted over the entire AIP as well as over user-defined AIP sectors. Each sector is described by a sector angle, $\theta_{s}$, and an azimuth angle center, $\theta_{c}$. The sector angle describes the sector extent or the angle between the left and right bounding rays. The azimuth angle describes the sector orientation or the angle between the sector bisector ray and the ray corresponding to the $\theta=0^{\circ}$ azimuth angle. To determine the sector with the lowest integrated total pressure, $\bar{P}_{t, \theta_{s}}$, the azimuth angle is changed by a user-specified increment. The increment is arbitrary and need not be positive, nor a factor of $360^{\circ}$.

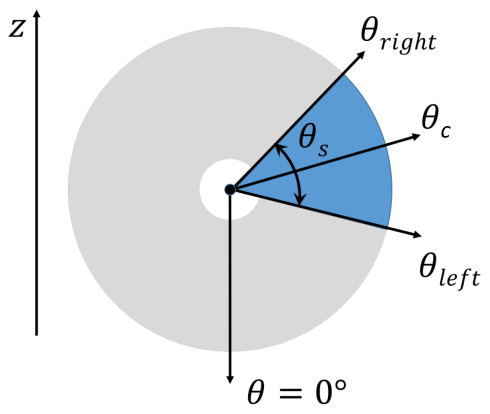

Figure 1. Illustration of angles in AIP.

Depending on the computational model, the AIP may or may not intersect an engine hub. As a result, the AIP can be shaped as a circular disk bounded by one outer curve or as a ring bracketed by two disjoint inner and outer curves. The computational grid may also include symmetry planes intersecting the AIP. In such cases, the current formulation requires the AIP to be symmetric and orthogonal to these planes. Prior to the simulation, all points belonging to symmetry planes are forced to lie in the appropriate plane.

The first step in computing the objective function is the identification of the AIP perimeter. The perimeter of the AIP is an ordered set of circumferential edges, where each edge is uniquely defined by a pair of grid points. Care is taken at partition boundaries, where endpoints of an edge may be assigned to different processors. Once the AIP perimeter has been established, all perimeter edges are sent to a single processor for convenience; the modest amount of data describing the AIP perimeter ensures this approach is feasible.

The current formulation requires a circular AIP. The center of the AIP can be identified from any three points located on the same perimeter segment. If the grid points do not lie on the analytic definition of a circle, variations in the calculated center position may occur if different sets of points are used. Care is taken to ensure that the same AIP center is identified regardless of the domain decomposition.

Since there are no restrictions on the orientation of the AIP, slight variations may occur between the directions of the normal vectors associated with each grid face comprising the AIP. To uniquely define the normal to the AIP, a unique boundary face is identified regardless of the domain decomposition. If the selected face is triangular, the AIP normal is computed as the cross product of the vectors forming the face. If the face is quadrilateral, then the AIP normal is computed as the cross product of the diagonal vectors in the face.

The next step is to identify the direction corresponding to $\theta=0^{\circ}$. Traditionally, this direction is perceived as pointing "down". To preserve both the usual orientations of the Cartesian axes and the convention of the $\theta=0^{\circ}$ direction, a reference vector has been defined as a unit vector aligned with the positive z-direction ("up") and the $\theta=0^{\circ}$ vector is defined as a unit vector that is $i$ ) orthogonal to the AIP normal and $i i$ ) has the greatest negative projection on the reference vector. Figure 1 illustrates the orientation of the $\theta=0^{\circ}$ vector.

To evaluate the objective function, the integrals of pressure appearing in Eq. 1 are computed over the entire AIP and a series of AIP sectors centered at $\theta_{c}$. The integration over the entire AIP area is performed as the summation of contributions from all grid faces in the AIP. The integrated quantity is defined at the grid points. To approximate the integral of a mass weighted quantity, the quantity is multiplied by the mass flow computed at that location. A second-order approximation of the integral over a triangular face is computed as the average of the quantities at the grid points multiplied by the face area. The integral over a quadrilateral face is computed as a sum of integrals over two triangles.

An integral over an AIP sector is computed as the summation of integrals over each grid face that intersects the sector. Faces that are not entirely located within the sector contribute according to the fraction of area 
inside the sector. An overlap between a triangular face and the sector is detected based on comparison of the angles $\left(\theta_{\text {left }}\right.$ and $\left.\theta_{\text {right }}\right)$ of the rays defining the sector

$$
\theta_{\text {left }}=\theta_{c}-\frac{1}{2} \theta_{s}, \quad \theta_{\text {right }}=\theta_{c}+\frac{1}{2} \theta_{s}
$$

with the angular coordinates of the grid points defining the face. The angular coordinate of a point is defined as the angle between the $\theta=0^{\circ}$ vector and the vector directed from the AIP center to the point (see Fig. 1). The angles $\theta_{\text {left }}$ and $\theta_{\text {right }}$ are expected to satisfy

$$
-180^{\circ} \leq \theta_{\text {left }} \leq \theta_{\text {right }} \leq 180^{\circ}
$$

If $\theta_{\text {left }}$ becomes smaller than $-180^{\circ}$, or $\theta_{\text {right }}$ becomes greater than $180^{\circ}$, or either cross the symmetry plane, the sector is divided into two smaller sectors and the bounding angles of each sector are normalized by adding or subtracting $360^{\circ}$ to satisfy Eq. 3. If all three points forming a triangle have angular coordinates between $\theta_{\text {left }}$ and $\theta_{\text {right }}$, then this triangle contributes its entire integral to the sector integral. If only some of the angular coordinates are inside the sector, then this triangle contributes only a portion of its integral to the sector integral. If all of the angular coordinates are outside of the sector, then this triangle may either contribute a portion or nothing to the sector integral. Special care is taken for points that are located on the rays corresponding to $\theta_{\text {left }}$ and $\theta_{\text {right }}$. An additional case requiring special attention is when the AIP center lies inside a grid face, and particularly, when the center lies on an edge or a grid point.

To determine the portion of a grid face inside an AIP sector, intersections of the rays corresponding to $\theta_{\text {left }}$ and $\theta_{\text {right }}$ with the edges of the triangle are established and analyzed. There are hundreds of combinations that result in different intersections of a grid face and a sector. The treatment for each specific combination is individually implemented and verified. The final objective function is computed according to Eq. 1.

The optimization problem can be formulated as follows

$$
\text { Find: } \min _{\boldsymbol{D}} F(\boldsymbol{D}, \boldsymbol{X}, \boldsymbol{Q}), \text { subject to } \boldsymbol{R}(\boldsymbol{D}, \boldsymbol{X}, \boldsymbol{Q})=0 \text { and } \boldsymbol{G}(\boldsymbol{D}, \boldsymbol{X})=0
$$

Here $\boldsymbol{R}(\boldsymbol{D}, \boldsymbol{X}, \boldsymbol{Q})$ is the discrete residual vector of the flow equations that depends on the design variables, grid coordinates, and flow solution; $\boldsymbol{G}(\boldsymbol{D}, \boldsymbol{X})$ is the residual vector of the grid equations that depends on the design variables and grid coordinates. Here, the grid equations are based on the linear elasticity equations of solid mechanics as described in Ref. 15 and 16.

A Lagrangian is formed to compute the sensitivity of the objective function $F$ to the design parameters,

$$
\mathcal{L}=F(\boldsymbol{D}, \boldsymbol{X}, \boldsymbol{Q})+\boldsymbol{\Lambda}_{R}^{T} \boldsymbol{R}(\boldsymbol{D}, \boldsymbol{X}, \boldsymbol{Q})+\boldsymbol{\Lambda}_{G}^{T} \boldsymbol{G}(\boldsymbol{D}, \boldsymbol{X})
$$

Here, $\boldsymbol{\Lambda}_{R}$ is a vector of Lagrange multipliers corresponding to the flow equations and $\boldsymbol{\Lambda}_{G}$ is a vector of Lagrange multipliers corresponding to the grid equations. The Lagrangian is differentiated with respect to the design parameters,

$$
\frac{d \mathcal{L}}{\boldsymbol{D}}=\frac{\partial F}{\partial \boldsymbol{D}}+\boldsymbol{\Lambda}_{R}^{T} \frac{\partial \boldsymbol{R}}{\partial \boldsymbol{D}}+\boldsymbol{\Lambda}_{G}^{T} \frac{\partial \boldsymbol{G}}{\partial \boldsymbol{D}}+\left\{\frac{\partial F}{\partial \boldsymbol{X}}+\boldsymbol{\Lambda}_{R}^{T} \frac{\partial \boldsymbol{R}}{\partial \boldsymbol{X}}+\boldsymbol{\Lambda}_{G}^{T} \frac{\partial \boldsymbol{G}}{\partial \boldsymbol{X}}\right\} \frac{d \boldsymbol{X}}{d \boldsymbol{D}}+\left\{\frac{\partial F}{\partial \boldsymbol{Q}}+\boldsymbol{\Lambda}_{R}^{T} \frac{\partial \boldsymbol{R}}{\partial \boldsymbol{Q}}\right\} \frac{d \boldsymbol{Q}}{d \boldsymbol{D}}
$$

Equating the coefficients of $\frac{d \boldsymbol{X}}{d \boldsymbol{D}}$ and $\frac{d \boldsymbol{Q}}{d \boldsymbol{D}}$ to zero results in a set of adjoint equations for $\boldsymbol{\Lambda}_{R}$ and $\boldsymbol{\Lambda}_{G}$

$$
\begin{aligned}
& \frac{\partial F}{\partial \boldsymbol{Q}}+\boldsymbol{\Lambda}_{R}^{T} \frac{\partial \boldsymbol{R}}{\partial \boldsymbol{Q}}=0 \\
& \frac{\partial F}{\partial \boldsymbol{X}}+\boldsymbol{\Lambda}_{R}^{T} \frac{\partial \boldsymbol{R}}{\partial \boldsymbol{X}}+\boldsymbol{\Lambda}_{G}^{T} \frac{\partial \boldsymbol{G}}{\partial \boldsymbol{X}}=0
\end{aligned}
$$

With Lagrange multipliers satisfying Eqs. 7 and 8, the sensitivity of the objective function with respect to the design variables is computed as

$$
\frac{d \mathcal{L}}{d \boldsymbol{D}}=\frac{\partial F}{\partial \boldsymbol{D}}+\boldsymbol{\Lambda}_{R}^{T} \frac{\partial \boldsymbol{R}}{\partial \boldsymbol{D}}+\boldsymbol{\Lambda}_{G}^{T} \frac{\partial \boldsymbol{G}}{\partial \boldsymbol{D}}
$$

In this manner, sensitivities of an objective function with respect to an arbitrary number of design parameters may be computed at a cost equivalent to that of one analysis. The sensitivities, $\frac{\partial F}{\partial Q}$, are computed 
by manual differentiation of the discrete form of $F$. In the current application, it is assumed that the AIP surface grid is not changing during the design process; therefore,

$$
\frac{d F}{d \boldsymbol{X}}=0 \text { and } \frac{d F}{d \boldsymbol{D}}=0
$$

The gradient computed in Eq. 9 is used by the gradient-based optimization package described in Ref. 15 to drive the optimization problem in Eq. 4.

\section{Verification of Sensitivity Analysis}

To verify the accuracy of the discrete sensitivity analysis, a simulation is performed using the engine configuration shown in Fig. 2, which is based loosely on the CFM56 family of engines. ${ }^{17}$ Since Eq. 1 does not explicitly depend on viscous parameters, the corresponding term $\frac{\partial F}{\partial Q}$ introduced for this study may be verified using an inviscid approach. This does not preclude the use of viscous simulations in practice, as the contributions from the full RANS equations have been previously verified in Ref. 14.
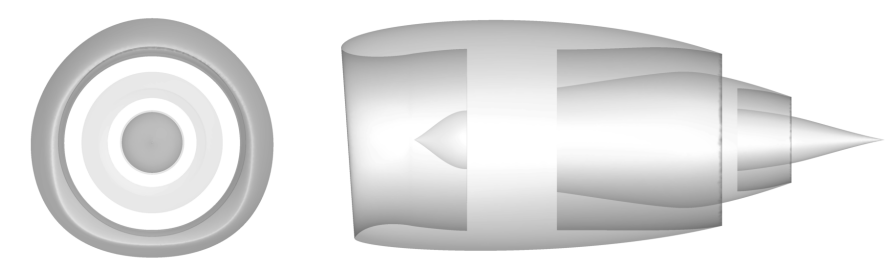

Figure 2. Engine configuration used for verification of sensitivity analysis.

For this test, the freestream Mach number is 0.8 and the angle of attack (AoA) is $2^{\circ}$. A static pressure boundary condition is used at the fan face corresponding to a local Mach number of 0.74 . The boundary condition representing bypass flow into the computational domain is subsonic with a ratio of the total pressure to the freestream static pressure equal to 2.52. The values of all solution quantities are prescribed for the engine core flow into the computational domain. Equation 1 is used as the objective function, with a sector angle $\theta_{s}=60^{\circ}$ and an azimuth angle increment of $10^{\circ}$.

To provide an independent verification of the adjoint-based implementation, comparisons are made with an approach based on the use of complex variables. ${ }^{18-20}$ The complex variable approach provides true secondorder accuracy for discrete sensitivity analyses while avoiding subtraction errors associated with real-valued finite difference approaches. For the results presented here, all equation sets have been converged to machine precision, including the real- and complex-valued grid and flow equations, as well as the adjoint equations. The perturbation size chosen for the complex-valued simulations is $10^{-50}$.

Sensitivities of the objective function for the current test case are shown in Table 1 for design variables corresponding to the freestream angle of attack as well as parameters representing the deflection of a mesh point on the inner surface of the lower engine cowl just upstream of the fan face. Excellent agreement is achieved between the adjoint implementation and the complex variable formulation for all design variables.

Table 1. Verification of sensitivities for flow distortion objection function.

\begin{tabular}{|l|c|c|c|c|}
\hline & AoA-derivatives & x-derivatives & y-derivatives & z-derivatives \\
\hline \hline Adjoint & 0.002092529448028 & 0.0001294071726387 & -0.000027863444220 & -0.000275093173340 \\
Complex & 0.002092529448028 & 0.0001294071726387 & -0.000027863444220 & -0.000275093173340 \\
\hline
\end{tabular}

\section{Demonstration of Mesh Adaptation and Design Optimization Capability}

This section contains three subsections: $i$ ) a demonstration of the mesh adaptation capability to reduce the error in the fan distortion calculation, ii) a verification case of the design optimization capability with a 
simplified geometry in freestream, and iii) a demonstration of the design optimization capability with a BLI geometry. A common ducted fan geometry is used for cases $i$ and $i$. This ducted fan is then resized and fitted as an aft fuselage propulsor to the BLI geometry that is used in case iii.

The ducted fan propulsion model for all cases is based on simple 1D thermodynamic principles, and mass flow is used as an input to control thrust. The inflow and outflow conditions for the fan are located at the fan face (i.e., the AIP) and fan exit, respectively. The inflow condition specifies the ratio of the static pressure at the AIP to the freestream static pressure, while the outflow condition specifies the ratio of the total pressure and the total temperature to the freestream static pressure and the static temperature, respectively. Here, the inflow boundary is a surface where mass flow exits the CFD domain and enters the engine, while the outflow boundary is the surface where mass flow exits the engine.

The CFD domains for all cases are meshed with AFLR3 ${ }^{21}$ and the first cell height for the viscous meshes is set to $y^{+}=1$. The FUN3D RANS analysis uses the Spalart-Allmaras turbulence model. The flow distortion metric is calculated through a mass-average of the CFD solution at the AIP (inflow face) using a $60^{\circ}$ sector angle $\left(D C_{60}\right)$. The flight conditions for all cases is Mach of 0.8 , altitude of $21,500 \mathrm{ft}$, AoA of $2^{\circ}$, and Reynolds number of 3 million per foot. Note that only non-dimensional total pressure $\left(P_{t}^{*}\right)$ is presented in this section. Dimensional total pressure can be calculated as $P_{t}=\rho_{\infty} a_{\infty}^{2} P_{t}^{*}$, where $\rho_{\infty}$ and $a_{\infty}$ are freestream density and speed of sound, respectively.

\section{A. Mesh Adaptation}

The verification case for the adjoint-based mesh adaptation process is a ducted fan-only model with a mass flow of $550 \mathrm{lbm} / \mathrm{s}$, as shown in Fig. 3. The flow area at the AIP and fan exit are $21.7 \mathrm{ft}^{2}$ and $18.8 \mathrm{ft}^{2}$, respectively. A static pressure ratio of 1.23 is specified at the AIP. The respective total pressure and total temperature ratios of 2.21 and 1.26 are specified at the fan exit.

The mesh adaptation process is initiated with an AFRL3 symmetric half mesh consisting of 36.5 million tetrahedral cells. The initial mesh is purposely over-refined to better capture the physics of this problem; the same mesh is used for the design verification case $i i$ that follows.

A total of 5 adaptation cycles are conducted with maximum element anisotropy restriction of 10 . The mesh adaptation objective is the reduction of the error in the fan distortion calculation. The error estimate and fan distortion $\left(D C_{60}\right)$ history for each adaptation cycle is provided in Fig. 4 . Note that after 5 iterations, the distortion value has increased by $90 \%$ of the initial value. This highlights the importance of the mesh adaptation capability when calculating fan distortion.

Despite not being fully converged, the adaptation was terminated after 5 cycles due to the high computational requirements needed to continue the mesh adaptation process. A comparison of the initial and adapted mesh at iteration 5 consisting of 241 million tetrahedral cells is provided in Fig. 5 . This figure shows the expected mesh refinement predominantly upstream of the ducted fan inflow.

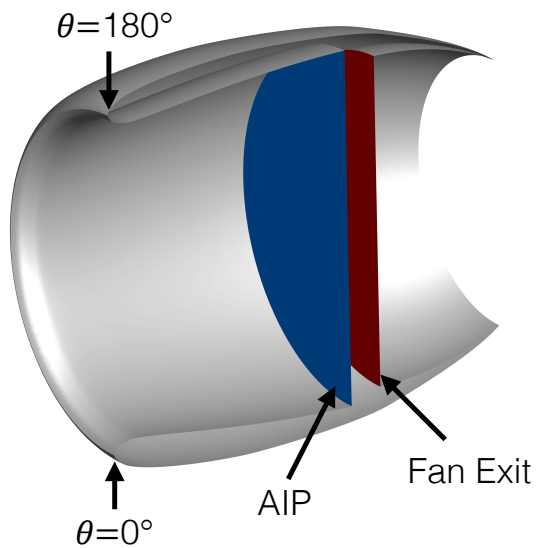

Figure 3. Geometry of ducted fan model.

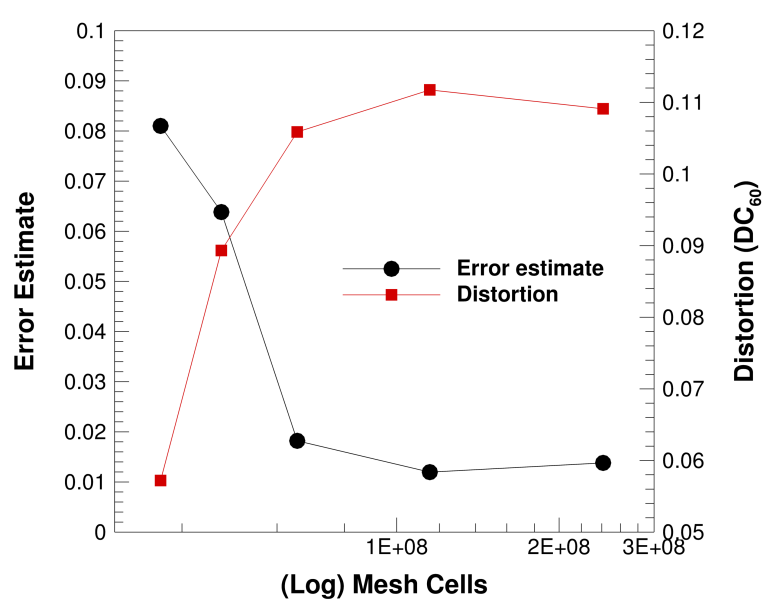

Figure 4. Error and fan distortion history for mesh adaptation process. 

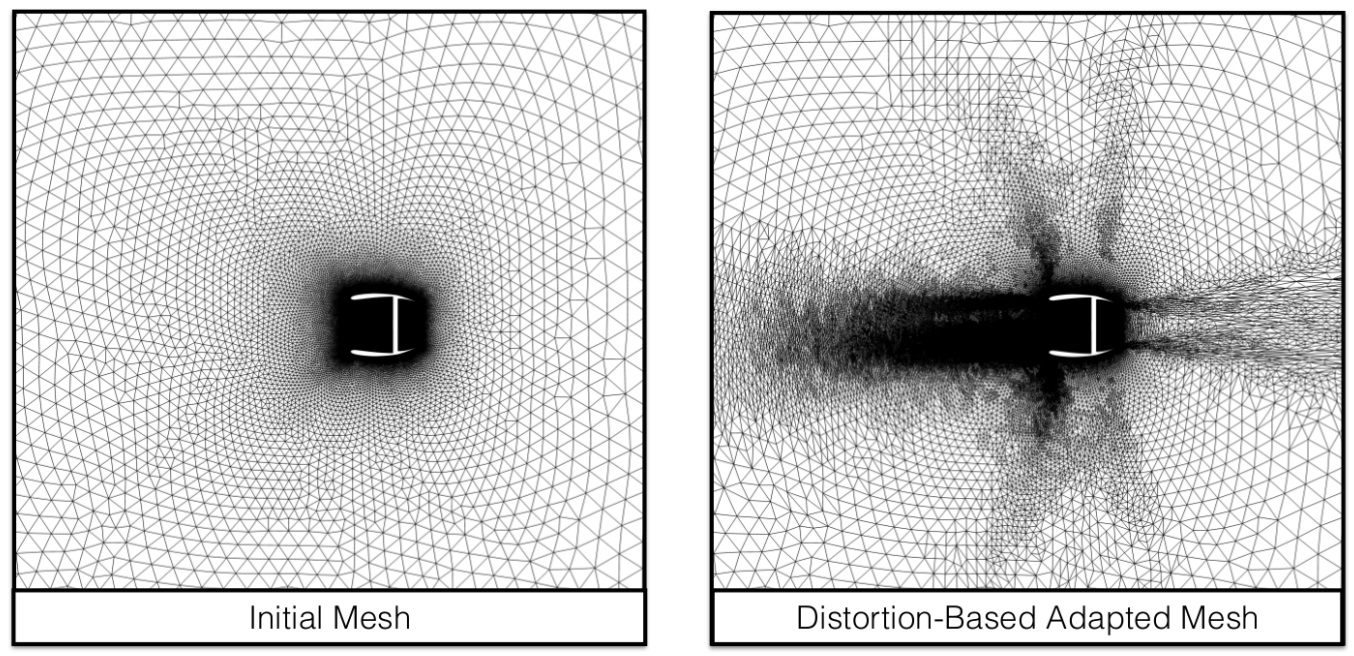

Figure 5. Comparison of initial and final adapted (iteration 5) symmetry mesh.

\section{B. Verification of Design Optimization}

An initial verification of the design optimization capability was conducted using the previous ducted fan model (Fig. 3). Ideally, the mesh used for the design optimization should be adapted to reduce the error in the distortion calculation, as shown in the previous section. For simplicity, the design optimization presented here is performed on the initial non-adapted mesh consisting of 36.5 million tetrahedral cells to reduce the computational time of each analysis.

The free-form shape deformation tool in Ref. 22 is used to parameterize and deform the discrete outermold-line (OML) during optimization. The design variables allow mesh deformation normal to the surface. The inlet geometry is parameterized using 5 equally spaced sections along the longitudinal axis with 4 design variables per section. The 4 design variables in each section are equally spaced in the $u$ - $v$ space along the azimuthal direction with the first variable in the section located at $\theta=0^{\circ}$ and the fourth variable located at $\theta=180^{\circ}$ (geometry is symmetric about the $x-z$ plane). The assumption given in Eq. 10 is satisfied by holding the intersection of the inlet with the AIP boundary constant. The gradient-based Sparse Nonlinear OPTimizer (SNOPT) ${ }^{23}$ is used to drive the optimization problem defined in Eq. 4.

The total pressure distribution along the azimuthal direction on the AIP is provided in Fig. 6(a) for the baseline and select design iterations. As expected for this simple axisymmetric geometry at an AoA of $2^{\circ}$, the worst sector (lowest total pressure) is centered along $\theta=0^{\circ}$. The distortion value $\left(D C_{60}\right)$ calculated for the final design (iteration 8) is 0.0018 , which corresponds to approximately $97 \%$ reduction in fan distortion. Note that for this simple problem the fan distortion has been significantly improved by design iteration 5 . Further comparison of the total pressure contours on the AIP between the baseline (left side) and shaped design at iteration 8 (right side) is shown in Fig. 6(b). This figure also shows that while there is significant improvement in circumferential distortion, variation in total pressure along the radial direction still exists. This radial distortion can be addressed by the appropriate use of blade twist.

\section{Design Optimization of BLI Geometry}

The geometry used to demonstrate the design capability in the presence of BLI is borrowed from an inhouse business jet concept referred to as the modular testbed aircraft or MTA450 (loosely based on the Gulfstream G450) and developed by Karl A. Geiselhart (NASA LaRC). The MTA450 design effort was part of a Convergent Aeronautic Solutions (CAS) X-plane study under the Transformative Aeronautics Concepts Program (TACP) at NASA. The vehicle concept is approximately $87 \mathrm{ft}$ in length, has a wingspan of $70 \mathrm{ft}$, and is equipped with an aft fuselage propulsor, as shown in the upper right of Fig. 7. The MTA450 was designed for steady and level cruise of Mach 0.8, at an altitude of 21,500 ft, and AoA of $2^{\circ}$.

The fan distortion of the aft fuselage propulsor for the MTA450 configuration is dominated by the wake of the vertical tail at $\theta=180^{\circ}$. Optimization of the MTA450 requires the parameterization of the vertical 


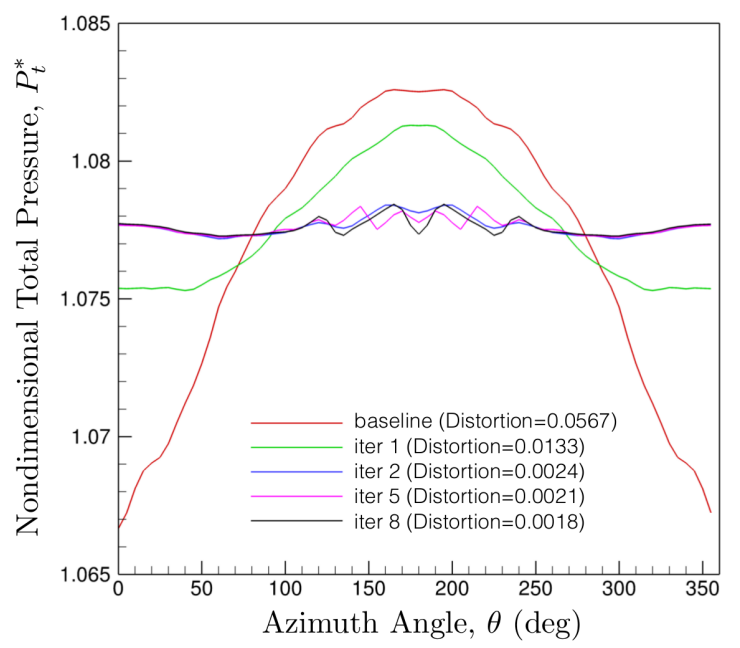

(a) Optimization history of the azimuthal distribution of total pressure.

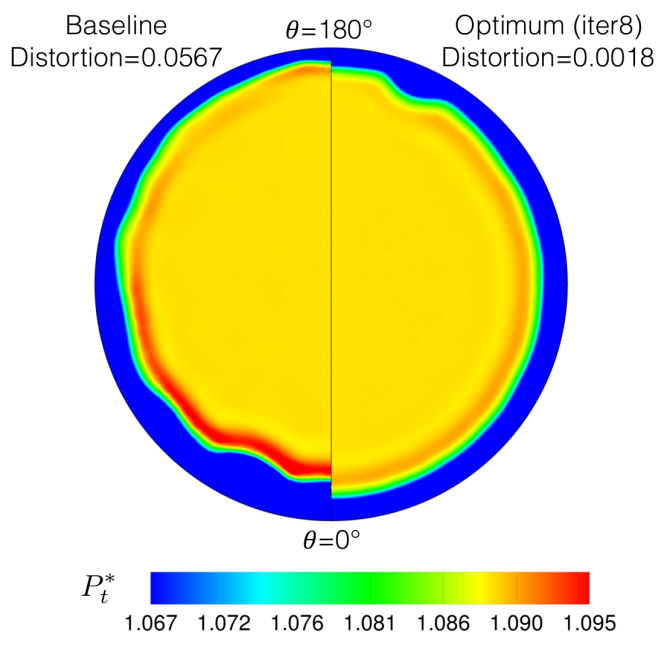

(b) Comparison of total pressure contours.

Figure 6. Comparison of total pressure between the baseline and shaped ducted fan geometry at the AIP.

tail and its intersection with the aft region of the fuselage. In an effort to simplify the complexity of the BLI problem and the parameterization of the geometry, only the fuselage and aft fuselage propulsor is retained from the original MTA450 model.

The same parameterization tool from case $i i$ is used to deform the discrete OML, and SNOPT is used to drive the optimization problem. Two parametric patches are defined for the inlet and aft fuselage region as denoted by the magenta color in Fig. 7. The parameterization for the inlet is the same used in the previous design case for the ducted fan in freestream consisting of 20 design variables. The parametric patch for the aft fuselage region consists of 5 equally spaced sections along the longitudinal direction, and 10 design variables per section. The 10 design variables in each section are equally spaced in the $u$ - $v$ space along the azimuthal direction with the first variable in the section located at $\theta=0^{\circ}$ and the tenth variable located at $\theta=180^{\circ}$ (geometry is symmetric about the $x$ - $z$ plane). The assumption given in Eq. 10 is satisfied by holding the intersections of the fuselage and inlet with the AIP boundary constant.

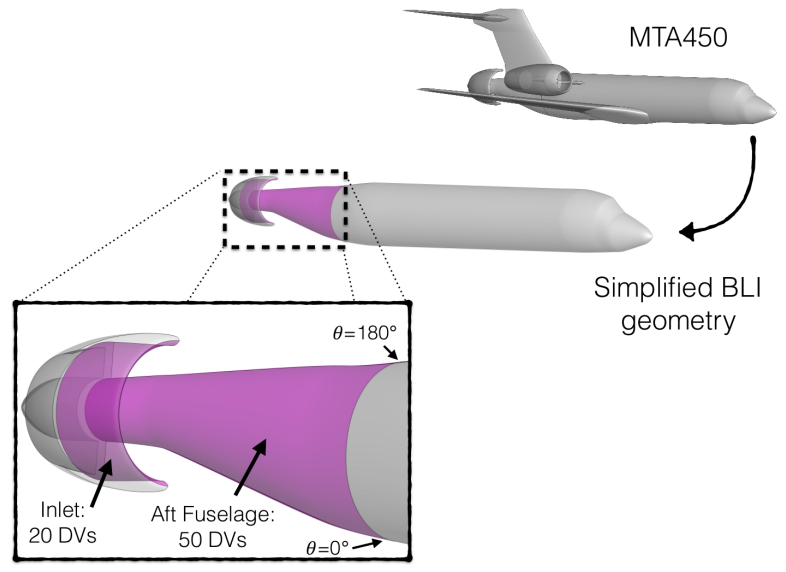

Figure 7. Parameterized surfaces of BLI geometry.

A fan propulsion model (described in Table 2) is used here to model the aft fuselage propulsor (a ducted fan). The ducted fan has been set to operate at a mass flow of $450 \mathrm{lbm} / \mathrm{s}$ and with an estimated gross thrust of 12,870 lbf. Aero-propulsive analysis iterations were performed between the CFD and propulsion analysis models. This allows a more accurate capture of the freestream inflow properties that are input to the fan propulsion model. The analysis convergence has been established using inlet pressure recovery $\left(P_{r}\right)$ as a 
feedback variable between the propulsion and aerodynamic analysis. An initial guess for $P_{r}$ is input into the propulsion model which computes the CFD boundary conditions at the AIP and fan exit. An updated $P_{r}$ value is then calculated from the CFD solution and this value is passed to the propulsion model. This process is repeated until an accepted tolerance in $P_{r}$ is achieved. For this particular case, the converged value of $P_{r}$ for a mass flow of $450 \mathrm{lbm} / \mathrm{s}$ is 0.87 , and the static pressure ratio at the AIP boundary condition is 1.127. The boundary condition at the fan exit is specified as a non-dimensional mass flow of $5.654(450 \mathrm{lbm} / \mathrm{s})$. Dimensional mass flow can be calculated by multiplying the non-dimensional mass flow by $\rho_{\infty} a_{\infty}$, where $\rho_{\infty}$ and $a_{\infty}$ are freestream density and speed of sound, respectively. Note that the flow properties specified at the boundary conditions of the AIP and fan exit are not varied during the design optimization. This approximation is acceptable for small geometry changes; however, the boundary conditions will need to be updated for larger changes in OML, which have a more significant impact on the performance of the aft fuselage propulsor.

Table 2. Specifications for the MTA450 BLI propulsor.

\begin{tabular}{|l|c|c|}
\hline Parameter & Value & Units \\
\hline \hline Mass flow & 450 & $\mathrm{lbm} / \mathrm{s}$ \\
Fan face (AIP) area & 16.7 & $\mathrm{ft}^{2}$ \\
Fan exit area & 13.7 & $\mathrm{ft}^{2}$ \\
Fan pressure ratio & 1.48 & \\
Fan adiabatic efficiency & 0.8945 & \\
Nozzle gross thrust coefficient & 0.9936 & \\
\hline
\end{tabular}

The optimization was conducted using 516 processor cores of a dual socket hex core $2.80 \mathrm{GHz}$ Intel X5660 Westmere computer cluster (43 nodes with 12 cores per node), and a non-adapted symmetric half mesh consisting of 21 million tetrahedral cells. The computational time for each CFD solution and adjoint calculation was approximately 15 minutes, and the total wall time for the entire optimization (13 design iterations) was approximately 7 hours.

The distribution of total pressure integrated along the azimuthal direction for the baseline and optimum design is provided in Fig. 8(a). Note that the sector with greatest flow distortion occurs at $\theta=0^{\circ}$ and after 4 design iterations ( 2 hours wall time), the distortion objective has been reduced by one order of magnitude to 0.0351 . The distortion for the final design solution (iteration 13) has been reduced by approximately $96 \%$ to a value of 0.0182. A comparison of the total pressure contours on the AIP between the baseline (left side) and shaped design at iteration 13 (right side) is shown in Fig. 8(b).

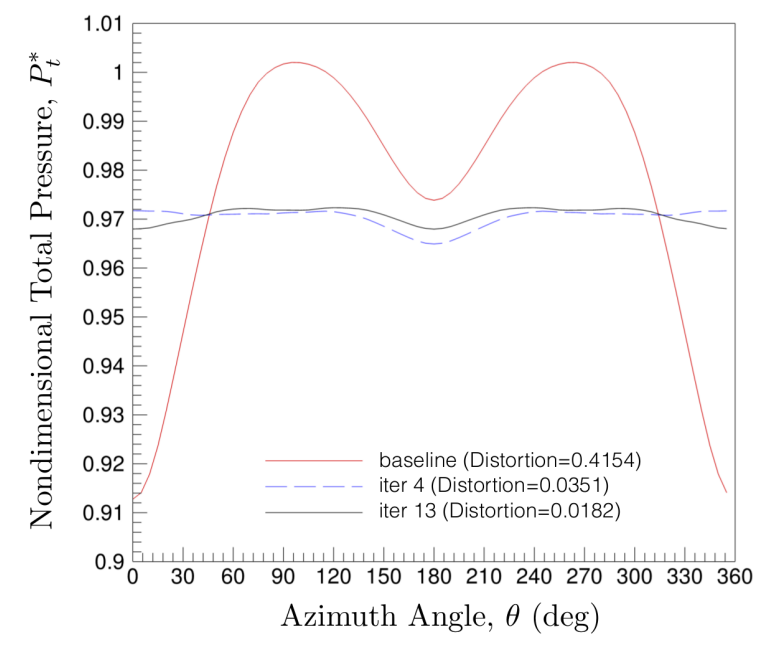

(a) Optimization history of the azimuthal distribution of total pressure.

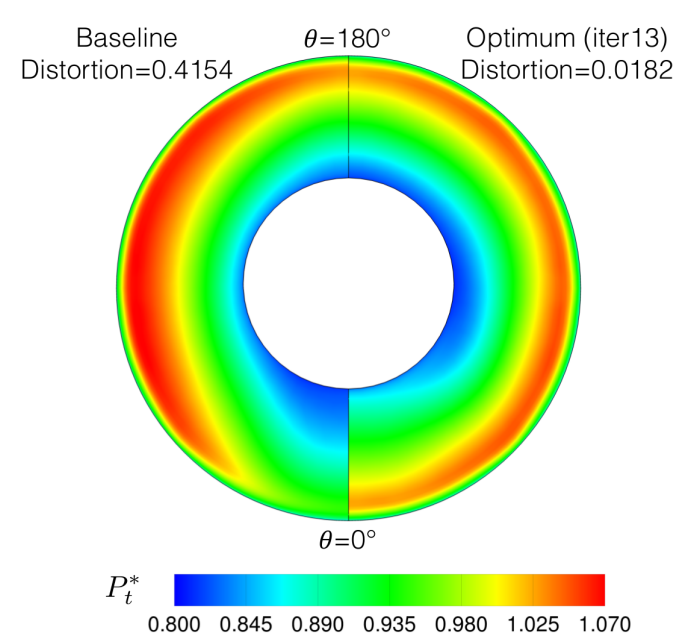

(b) Comparison of total pressure contours.

Figure 8. Comparison of total pressure between the baseline and shaped BLI geometry at the AIP. 
A comparison of the baseline and shaped geometry (iteration 13) is provided in Fig. 9(a). The plot in Fig. 9(b) shows the normal displacement for the optimized geometry relative to the baseline geometry. The maximum displacement of the shaped geometry is 6.3 in and occurs at approximately $\theta=90^{\circ}$ in the aft fuselage region. The maximum displacement of the inlet is 0.42 in and occurs at $\theta=0^{\circ}$.

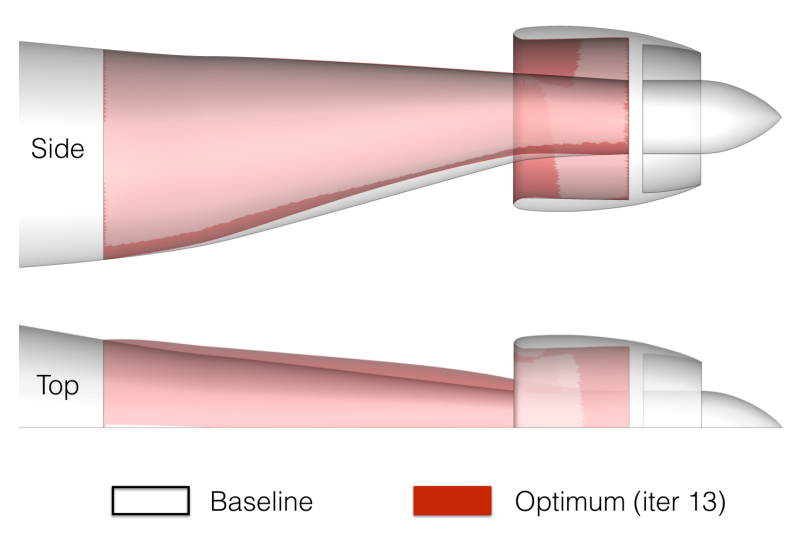

(a) Geometry comparison.

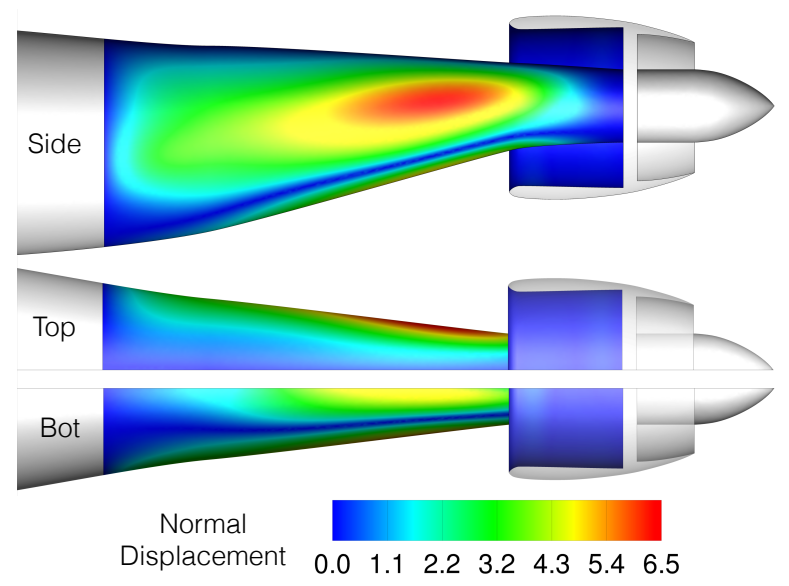

(b) Displacement from baseline (in).

Figure 9. Comparison of baseline and optimized aft fuselage geometry.

For reference, aerodynamic and propulsive performance were also tracked despite not being part of the optimization objective. A comparison of select aerodynamic and propulsion performance metrics for the baseline and optimized geometry is given in Table 3. Note that despite significant improvement in flow distortion, inlet total pressure recovery remains relatively unchanged, within $1 \%$ of the baseline. Degradation of the aerodynamic performance (i.e., pressure- and viscous-based drag force) for the optimized geometry is less than $1 \%$ for the entire geometry. The largest percentage reduction in drag force of $40 \%$ is observed at the inlet, which results in a reduction of $211 \mathrm{lbf}$ in ram drag. However, this reduction has been offset by an increase in drag force of approximately $219 \mathrm{lbf}$ for the nacelle cowl. The drag force contribution of the parameterized aft fuselage increased by $216 \mathrm{lbf}$ but has also been offset by a decrease of $204 \mathrm{lbf}$ in drag contribution from the main fuselage. The significant improvement in fan distortion with minimal degradation in aerodynamic performance indicates a possibility to simultaneously improve aerodynamic performance and fan distortion by incorporating aerodynamic performance into the optimization objective.

Table 3. Comparison of aerodynamic and propulsion performance.

\begin{tabular}{|l|c|c|c|}
\hline Parameter & Baseline & Optimum (iter 13) & Change \\
\hline \hline Distortion $\left(D C_{60}\right)$ & 0.4154 & 0.0182 & $-95.6 \%$ \\
Worst distortion sector $(\mathrm{deg})$ & 348 & 276 & \\
Pressure recovery & 0.87 & 0.86 & $-1.1 \%$ \\
\hline Drag force from fuselage and nacelle cowl (lbf) & 1,584 & 1,815 & $14.5 \%$ \\
Drag force from fuselage, nacelle, inlet, and nozzle (lbf) & 3,912 & 3,936 & $0.62 \%$ \\
\hline
\end{tabular}

\section{Summary}

A discrete adjoint-based approach for design optimization with a fan distortion objective was formulated, and three demonstration cases were presented. The implemented distortion calculation is based on the areaor mass-average of flow properties for all grid faces that lie on the aerodynamic interface plane (AIP). The first case demonstrated the adjoint-based mesh adaptation capability to reduce the error in the distortion calculation. The second and third case demonstrated the design optimization capability to reduce distortion at the AIP of a ducted fan in freestream and under boundary layer ingestion (BLI) conditions, respectively. 
Both design cases successfully reduced the distortion by an order of magnitude within 2 hours of wall time. The design optimization of the BLI geometry showed that it may also be possible to mitigate fan distortion without adverse effects to aerodynamic and propulsive efficiency.

Future work includes the application of the new design capability to a full configuration. Of particular interest are BLI concepts with vertical tails upstream of the aft fuselage fan, which exhibit significant flow distortion at $\theta=180^{\circ}$ and require a more sophisticated parameterization scheme of the aft fuselage region and vertical tail. It is possible that more complex design problems, which result in larger geometrical changes, may require additional iterations of the aero-propulsive analyses. It is also important to understand how geometries that are optimized to reduce fan distortion at a cruise condition affect aerodynamic and propulsive performance during takeoff and throughout the entire cruise segment. Therefore, a multipoint design sensitivity study should be conducted to better quantify the robustness of the design solution to variation in flight conditions.

\section{Acknowledgment}

This work was supported by the Advanced Air Transport Technology Project under the NASA Advanced Air Vehicles Program. The authors would like to acknowledge Karl A. Geiselhart at NASA LaRC for discussions and recommendations related to propulsion modeling, and for providing a fan propulsion model and the modular testbed configuration (MTA450) with an integrated BLI ducted fan that was used as part of the design demonstration case. The authors would also like to thank Jan-Reneé Carlson at NASA LaRC for discussions associated with the application of FUN3D engine boundary conditions, particularly at the AIP of the ducted fan used for the BLI design case.

\section{References}

\footnotetext{
${ }^{1}$ Grose, T. K., "Reshaping Flight for Fuel Efficiency: Five Technologies on the Runway," http://news. nationalgeographic.com/news/energy/2013/04/130423-reshaping-flight-for-fuel-efficiency/ [cited April 2017], April 2013.

${ }^{2}$ Moody, E., "Focus On Fuel Savings," http://aviationweek.com/awin/focus-fuel-savings [cited April 2017 ], May 2012.

${ }^{3}$ Collier, F. and Wahls, R., "ARMD Strategic Thrust 3: Ultra-Efficient Commercial Vehicles Subsonic Transport," https: //www.hq.nasa.gov/office/aero/pdf/ARMD-SIP-Thrust-3a-508.pdf [cited April 2017], May 2016.

${ }^{4}$ Nagel, A. L., Alford Jr., W. J., and Dugan Jr., J. F., "Future Long-Range Transport - Prospects for Improved Fuel Efficiency," AIAA Paper 75-316, March 1975.

${ }^{5}$ Plas, A. P., Sargeant, M. A., Madani, V., Crichton, D., Greitzer, E. M., Hynes, T. P., and Hall, C. A., "Performance of a Boundary Layer Ingesting (BLI) Propulsion System," AIAA Paper 2007-450, January 2007.

6 "Performance Analysis of Wake and Boundary-Layer Ingestion for Aircraft Design," Journal of Aircraft, Vol. 53, No. 5, 2016, pp. 1517-1526.

${ }^{7}$ Gissen, A. N., Vukasinovic, B., McMillan, M. L., and Glezer, A., "Distortion Management in a Boundary Layer Ingestion Inlet Diffuser Using Hybrid Flow Control," Journal of Propulsion and Power, Vol. 30, No. 3, 2014, pp. 834-844.

${ }^{8}$ Rodriguez, D. L., "Multidisciplinary Optimization Method for Designing Boundary-Layer-Ingesting Inlets," Journal of Aircraft, Vol. 46, No. 3, 2009, pp. 883-894.

${ }^{9}$ Lee, B. J., Liou, M.-S., and Kim, C., "Optimizing a Boundary-Layer-Layer Ingestion Inlet by Discrete Adjoint Approach," AIAA Journal, Vol. 48, No. 9, 2010, pp. 2008-2016.

${ }^{10}$ Anonymous, "Gas Turbine Engine Inlet Flow Distortion," SAE ARP-1420, Society of Automotive Engineers, March 1978.

11 "FUN3D Resource Website," http://fun3d.larc.nasa.gov [cited April 2017].

12 van Leer, B., "Multidisciplinary Optimization Method for Designing Boundary-Layer-Ingesting Inlets," Journal of Computational Physics, Vol. 32, 1979, pp. 101-136.

${ }^{13}$ Biedron, R. T., Jan-Reneé Carlson, J. M. D., Gnoffo, P. A., Hammond, D. P., Jones, W. T., Kleb, B., Lee-Rausch, E. M., Nielsen, E. J., Park, M. A., Rumsey, C. L., Thomas, J. L., , and Wood, W. A., "FUN3D Manual 13.1," NASA TM-2017-19580, 2017.

${ }^{14}$ Nielsen, E. J. and Diskin, B., "Discrete Adjoint-Based Design for Unsteady Turbulent Flows on Dynamic Overset Unstructured Grids," AIAA Journal, Vol. 51, No. 6, 2013, pp. 1355-1373.

${ }^{15}$ Nielsen, E. J. and Anderson, W. K., "Recent Improvements in Aerodynamic Design Optimization on Unstructured Meshes," AIAA Journal, Vol. 40, No. 6, 2002, pp. 1155-1163.

${ }^{16}$ Biedron, R. T. and Thomas, J. L., "Recent Enhancements to the FUN3D Flow Solver for Moving-Mesh Applications," AIAA Paper 2009-1360, January 2009.

${ }^{17}$ Bilien, J. and Matta, R., "The CFM56 Venture," AIAA Paper 1989-2038, July 1989.

${ }^{18}$ Lyness, J. N., "Numerical Algorithms Based on the Theory of Complex Variables," Proceedings of the 22nd ACM National Conference, Thomas Book Company, Washington, D.C., July 1967, pp. 124-134.

${ }^{19}$ Lyness, J. N. and Moler, C., "Numerical Differentiation of Analytic Functions," SIAM Journal on Numerical Analysis, Vol. 4, No. 2, 1967, pp. 202-210.
} 
${ }^{20}$ Anderson, W. K., Newman, J. C., Whitfield, D. L., and Nielsen, E. J., "Sensitivity Analysis for the NavierStokes Equations on Unstructured Meshes Using Complex Variables," AIAA Journal, Vol. 39, No. 1, 2001, pp. 56-63.

21 "AFLR Website," http://www.simcenter.msstate.edu/research/cavs_cfd/aflr.php [cited April 2017].

${ }^{22}$ Samareh, J. A., "Aerodynamic Shape Optimization based on free-form deformation," AIAA Paper 2004-4630, June 2004.

${ }^{23}$ Gill, P. E., Murray, W., and Saunders, M. A., "SNOPT: An SQP Algorithm for Large-Scale Constrained Optimization," SIAM Review, Vol. 47, No. 1, 2005, pp. 99-131. 